UNIVERSIDADE DE SÃO PAULO

ESCOLA DE EDUCAÇÃO FÍSICA E ESPORTE

INTERFERÊNCIA CONTEXTUAL NA APRENDIZAGEM DE HABILIDADES GRÁFICAS EM CRIANÇAS: ADAPTAÇÃO IPSI E CONTRALATERAL

Alexandre Gomide Frugiuele Babo

SÃO PAULO

2008 
INTERFERÊNCIA CONTEXTUAL NA APRENDIZAGEM DE HABILIDADES GRÁFICAS EM CRIANÇAS: ADAPTAÇÃO IPSI E CONTRALATERAL

\section{ALEXANDRE GOMIDE FRUGIUELE BABO}

Dissertação/Tese apresentada à Escola de Educação Física e Esporte da Universidade de São Paulo, como requisito parcial para obtenção do grau de Mestre em Educação Física. 
Babo, Alexandre Gomide Frugiuele Interferência contextual na aprendizagem de habilidades gráficas em crianças: adaptação ipsi e contralateral. Alexandre Gomide Frugiuele Babo. -- São Paulo: [s.n.], 2008.

ix, 29p.

Dissertação (Mestrado) - Escola de Educação Física e Esporte da Universidade de São Paulo.

Orientador: Prof. Dr. Luis Augusto Teixeira

1. Interferência Contextual 2. Transferância contralateral 3. Padrões gráficos. 


\section{AGRADECIMENTOS}

Ao Prof. Dr. Luis Augusto Teixeira, por orientar-me com sabedoria respeitando meus limites conduzindo-me magistralmente nos estudos e pesquisa. Aos meus familiares, Regina e Kaio, pelo amor demonstrado no decorrer de mais esta empreitada, pela paciência demonstrada durante os anos em que me dediquei aos estudos, pela compreensão e amor demonstrados em todos os momentos de nossa vida. A minha mãe, Rita, que acreditou e de tudo fez para que minha jornada se tornasse possível. Aos amigos do SHM que sempre me auxiliaram prontamente quando necessitei Victor, Leandro, Elke, Andréa, Raimundo e Carla. Aos Padres do Externato Salesiano Santa Teresinha pela confiança e investimento inicial em minha progressão continuada o que está culminando neste.

"Não sabendo que era impossível, ele foi lá e fez..."

$$
\text { (Lao - Tse) }
$$


SUMÁRIO

Página

$\begin{array}{ll}\text { LISTA DE FIGURAS } & \text { iii }\end{array}$

LISTA DE SIGLAS, ABREVIAÇÕES E SÍMBOLOS iv

LISTA DE ANEXOS $\quad \mathrm{V}$

RESUMO vi

ABSTRACT vii

1 INTRODUÇÃO 1

2 REVISÃO DE LITERATURA 2

3 OBJETIVO

4 JUSTIFICATIVA 8

5 MÉTODO 9

5.1 Participantes 9

5.2 Tarefa e equipamentos 9

5.3 Delineamento experimental e procedimentos 10

5.4 Análise dos dados $\quad 12$

6 RESULTADOS 12

6.1 Tempo de movimento 12

6.1.1 Fase de aquisição 12

6.1.2 Retenção e transferência 13

6.2 Variabilidade de tempo de movimento 14

$\begin{array}{lll}\text { 6.2.1 } & \text { Fase de aquisição } & 14\end{array}$

6.3 Retenção e transferência 15

7 DISCUSSÃO 15

8 CONCLUSÃO 18

9 LIMITAÇÕES DO TRABALHO 18

10 SUGESTÕES PARA ESTUDOS FUTUROS 18 
REFERÊNCIAS BIBLIOGRÁFICAS

ANEXOS 


\section{LISTA DE FIGURAS}

Página

FIGURA 1 - Formas geométricas empregadas na fase de prática (círculo, cruz e 10 triângulo eqüilátero) e na fase de transferência (diamante).

FIGURA 2 - Média do TM a cada três tentativas no contorno das figuras geométricas durante a fase de AQ dos grupos AL/BA

FIGURA 3 - Média do TM a cada três tentativas no contorno das figuras geométricas durante a fase RT/TRd/Ter, grupos AL/BA/CO

FIGURA 4 - Média da variabilidade do TM a cada três tentativas no contorno das 14 figuras círculo, cruz e triângulo durante a fase AQ, grupos AL/BA

FIGURA 5 - Média da Variabilidade do TM a cada três tentativas da fase de 15 RT/TRd/TRe, grupos AL/BA/CO 


\section{LISTA DE SIGLAS, ABREVIAÇÕES E SÍMBOLOS}

$\begin{array}{ll}\text { AL } & \text { Alta interferência contextual } \\ \text { AQ } & \text { Aquisição } \\ \text { BA } & \text { Baixa interferência contextual } \\ \text { CO } & \text { Controle } \\ \text { IC } & \text { Interferência contextual } \\ \text { RT } & \text { Retenção } \\ \text { TM } & \text { Tempo do movimento } \\ \text { TR } & \text { Transferência } \\ \text { TR } & \text { Transi } \\ \text { TR }_{\text {contra }} & \text { Transferência ipsilateral }\end{array}$




\section{LISTA DE ANEXOS}

Página

$\begin{array}{lll}\text { ANEXO I } & \text { - Termo de consentimento livre e esclarecido } & 25 \\ \text { ANEXO II } & \text { - Cronograma do experimento } & 27 \\ \text { ANEXO III } & \text { - Resultados da análise de variância de tempo de movimento } & 28 \\ \text { ANEXO IV } & \text { - Resultados da análise de variância de variabilidade tempo de } 29 \\ & & \\ & \end{array}$


RESUMO

\title{
INTERFERÊNCIA CONTEXTUAL NA APRENDIZAGEM DE HABILIDADES GRÁFICAS EM CRIANÇAS: ADAPTAÇÃO IPSI E CONTRALATERAL
}

\author{
Autor: ALEXANDRE GOMIDE FRUGIUELE BABO \\ Orientador: PROF. DR. LUIZ AUGUSTO TEIXEIRA
}

Em um estudo recente, Guadagnoli e Lee (2004) propuseram que o efeito de interferência contextual (IC) depende da dificuldade funcional de uma tarefa motora, com reversão do efeito de aprendizagem esperado quando a dificuldade imposta pela tarefa é elevada. No presente estudo esta hipótese foi testada em crianças na aprendizagem de uma tarefa de desenhos de padrões gráficos em uma base digitalizadora, recebendo feedback visual imediato invertido em um monitor de microcomputador. Foram avaliadas 30 crianças de 8-9 anos de idade, as quais foram designadas a três grupos: baixa IC, com prática variada entre blocos de tentativas possuindo o mesmo padrão gráfico; alta IC, com variação aleatorizada dos padrões gráficos entre as tentativas dentro de cada bloco; e controle, sem prática na tarefa. Após a etapa de prática, o tempo de movimento foi comparado entre os grupos em testes de retenção, transferência ipsilateral e transferência contralateral de aprendizagem. Os resultados mostraram que os dois grupos experimentais obtiveram resultados semelhantes de aprendizagem em todas as avaliações realizadas, com ambos os grupos apresentando resultados superiores ao grupo controle. A ausência de diferença entre os grupos experimentais indica que ambos os modos de variar a prática, sob baixa e alta IC, induziram efeitos semelhantes de aprendizagem, oferecendo apenas suporte parcial à hipótese de Guadagnoli e 
Lee. A vantagem dos grupos experimentais no teste de transferência contralateral indica que a adaptação devida à aprendizagem da tarefa não foi específica ao sistema neuromuscular empregado durante a prática.

Palavras-chave: interferência contextual; transferência contralateral; padrões gráficos. 


\begin{abstract}
CONTEXTUAL INTERFERENCE IN THE LEARNING OF GRAPHIC SKILLS IN CHILDREN: IPSI AND CONTRALATERAL ADAPTATION
\end{abstract}

Author: ALEXANDRE GOMIDE FRUGIUELE BABO

Adviser: PROF. DR. LUIS AUGUSTO TEIXEIRA

In a recent study, Guadagnoli and Lee (2004) proposed that the contextual interference (CI) effect depends on the functional difficulty of a motor task, with reversion of the expected learning effect when the difficulty imposed by a task is high. In the present study that hypothesis was tested in children in the learning of a task of drawing graphic patterns on a digitizing tablet while receiving immediate inversed visual feedback on a microcomputer monitor. Thirty 8-9 year-old children were assessed, who were assigned to three groups: low CI, with variability between blocks of trials composed of the same graphic pattern; high CI, with random variability within blocks of trials; and control, without practice on the task. Following the practice phase movement time was compared between the groups in retention, ipsilateral transfer, and contralateral transfer tests. Results showed that both experimental groups achieved similar results of learning in all tests, with the two groups presenting better results in comparison with the controls. Absence of difference between the experimental groups indicates that both modes of practice variability, under low and high CI, induced similar effects of learning, offering only partial support for the Guadagnoli and Lee's 
hypothesis. The advantage of the experimental groups over the controls in the contralateral transfer of learning indicates that adaptation was not specific to the neuromuscular system employed during practice.

Keywords: contextual interference; contralateral transfer; graphic patterns. 
A organização de um programa de prática a fim de promover a aprendizagem de habilidades motoras de forma mais efetiva tem sido foco de estudo na área de aprendizagem motora. Uma das variáveis que têm se revelado de grande importância nessa busca de potencialização dos efeitos da prática é a diversificação de forma aleatória entre variações do mesmo padrão de movimento ou de variações entre diferentes padrões motores em uma seqüência de tentativas. Esse efeito foi chamado de interferência contextual. O pioneiro nos estudos sobre este tema foi BATTIG (1966), que a partir de pesquisas em aprendizagem verbal verificou a superioridade da prática variada aleatoriamente em relação à prática variada em blocos de tentativas. SHEA e MORGAN (1979) ampliaram os achados de Battig, mostrando que um efeito semelhante ocorre na aprendizagem de habilidades motoras. Isto é, após prática em uma tarefa seqüencial de forma aleatória, um grupo de participantes obteve melhores resultados de retenção e transferência de aprendizagem em comparação a outro grupo que praticou a tarefa com variabilidade em blocos de tentativas. Este efeito tem sido corroborado em uma série de estudos subseqüentes (veja MAGILL \& HALL, 1990, para uma revisão).

Recentemente, no entanto, tem sido proposto que a alta interferência contextual seria benéfica somente em tarefas simples, mas não em tarefas complexas. Esta proposição foi feita por GUADAGNOLI e LEE (2004), a partir de uma ampla revisão dos estudos sobre o tema. Em linhas gerais, propõe-se que uma tarefa motora representa desafios específicos para indivíduos com diferentes níveis de habilidade. Para executantes habilidosos a alta interferência contextual seria benéfica, pois neste caso a demanda atencional de controle motor seria baixa, o que tornariam disponíveis recursos para lidar com a demanda atencional extra imposta pelo contexto aleatório. Aprendizes novatos, por outro lado, estão demasiadamente envolvidos com o controle de aspectos básicos de coordenação dos movimentos. Neste caso, a introdução de variações aleatorizadas traria uma sobrecarga de processamento demasiada para o aprendiz. A conseqüência de tal demanda exagerada de processamento seria um prejuízo ao invés de benefício para a aprendizagem da tarefa motora em comparação a um contexto com variações menos freqüentes da habilidade. Os princípios gerais da proposta apresentados por Guadagnoli e Lee são os seguintes: (a) a aprendizagem não pode ocorrer na ausência de informação, (b) a aprendizagem é retardada na presença de informação demasiada ou a aprendizagem é limitada quando a informação é reduzida em excesso, e (c) para ocorrer 
aprendizagem, há necessidade de uma quantidade ótima de informação, que varia de acordo com o nível de habilidade do indivíduo e a dificuldade do que vai ser aprendido.

De acordo com a conceituação formulada por GUADAGNOLI e LEE (2004), assim, deveria ser esperado um efeito contrário àquele esperado quando a alta interferência contextual é aplicada à aprendizagem de iniciantes em habilidades complexas. Além disso, VAN ROSSUN (1990) propõe que a prática com variabilidade aplicada a crianças deveria apresentar resultados mais evidentes, pois uma explicação possível é que estes não tenham um número suficiente de experiências motoras que lhes permitam responder bem a novas situações. Indivíduos adultos teriam mais experiências motoras ao longo da vida, o que levaria a representações mais estáveis na memória motora. A partir de tais noções, optou-se pela utilização de crianças como participantes do presente estudo, com avaliação da aprendizagem de uma tarefa envolvendo o controle de vários graus de liberdade. Além disso, a dificuldade da tarefa foi potencializada pela inversão do feedback visual. A partir deste arranjo experimental, assim, foi formulada a hipótese de que se a alta interferência contextual tiver um efeito deletério na aprendizagem de habilidades motoras complexas, a baixa interferência contextual deveria ser mais positiva nesta situação.

Outro ponto a ser considerado a respeito do efeito da interferência contextual é que a prática variada de forma aleatória gera melhor adaptação tanto quando a transferência é feita para uma variação do padrão de movimento praticado (VERA \& MONTILLA, 2003), como quando a transferência é feita para um padrão de movimento diferente (SMITH, GREGORY \& DAVIES, 2003). Estes resultados indicam que a adaptação ocorre em um nível mais abstrato de representação da ação na memória, relativamente independente do sistema neuromuscular empregado durante a prática. Desta forma, se houver um benefício da baixa interferência contextual em iniciantes em tarefas com alta dificuldade funcional é esperado que este benefício seja transferido também para o lado contralateral àquele empregado durante a aquisição da tarefa.

Desde a proposição da teoria de esquema (SCHMIDT, 1975), tem sido mostrado que a variabilidade de prática tem efeitos benéficos sobre a retenção e transferência de aprendizagem motora (SHEA \& WULF, 2005). A comparação de diferentes formas de variar a 
prática tem revelado, ainda, que a aprendizagem pode ser potencializada em situações de alta variabilidade intertentativas. Isto é, quando se comparam situações em que tentativas com mesmas características são concentradas em blocos sem variabilidade interna versus variação de suas características a cada tentativa de forma aleatorizada, tem sido observado que a segunda induz melhor retenção e transferência de aprendizagem (MAGILL \& HALL, 1990). Esse efeito ficou conhecido como interferência contextual.

BATTIG (1966) foi o primeiro a identificar este efeito em seus estudos sobre aprendizagem verbal. O fenômeno da interferência contextual refere-se a achados consistentes de que praticando diversas tarefas relacionadas em ordem aleatória, definido como alta interferência contextual, resulta em desempenho inferior durante a aquisição. No entanto esse procedimento aumenta a retenção e transferência quando comparado à prática em bloco. Inversamente, onde as tarefas são praticadas em blocos de tentativas, ou baixa interferência contextual, o desempenho durante a prática é melhor enquanto a retenção e transferência da habilidade são inferiores à prática aleatória. Desde que SHEA e MORGAN (1979) publicaram um experimento pioneiro sobre interferência contextual na aprendizagem de habilidades motoras, um extensivo corpo de pesquisa tem sido produzido sobre este tópico (BRADY, 2004; MAGILL \& HALL, 1990, para revisões). Recentemente, o efeito da interferência contextual tem sido demonstrado em uma variedade de tarefas na área de aprendizagem motora. Estas tarefas incluem a manipulação do timing coincidente através da pressão de teclas de computador (WRIGHT, BLACK, BRUCKNER \& MAGNUSON, 2004; WRIGHT, MAGNUSON \& BLACK, 2005), arremesso (VERA \& MONTILLA, 2003), chute (LI \& LIMA, 2002; WEGMAN, 1999), interceptação de alvos móveis (BABO, AZEVEDO NETO \& TEIXEIRA, 2008) e escritas (MASLOVAT CHUA, LEE \& FRANCS, 2004; STE-MARIE, CLARK, FINDLAY \& LATIMER, 2004).

As duas explanações predominantes para o efeito da IC são as hipóteses da elaboração do plano de ação (SHEA \& ZIMNY, 1983) e a hipótese do esquecimento e reconstrução do plano de ação (LEE \& MAGILL, 1985). De acordo com a hipótese de elaboração, ao desempenhar uma tarefa através de um programa de prática aleatória o indivíduo se engaja em um processo de aprendizagem mais elaborado e distintivo, pois existe a presença simultânea de itens na memória de trabalho. Este processo, de acordo com Shea e Zimny, facilita a identificação da quantidade de similaridades e diferenças entre os itens, redundando em melhor representação na memória. BATTIG (1979) afirmou que o efeito facilitador da prática 
aleatória ocorre devido o aumento no envolvimento cognitivo e a quantidade de processamento, que ele considerou ser a causa dos efeitos na memória de longo prazo. Em contraste, em um regime de prática em blocos somente um item reside na memória de trabalho, impedindo um processamento adicional e mais profundo (melhor codificação) e consequentemente uma aprendizagem mais pobre. Apesar de reconhecerem que e a aprendizagem motora e aprendizagem verbal são bem diferentes uma da outra, Shea e Zimny afirmaram que ambas as tarefas seriam conceituadas como de processamento cognitivo.

A segunda hipótese, reconstrução do plano de ação (LEE \& MAGILL, 1983), baseia-se na proposição de que a alta interferência contextual requer que o plano de ação elaborado para desempenhar uma determinada tarefa seja esquecido, para que um novo plano seja elaborado para lidar com as variações dessa tarefa, em um processo contínuo de reconstrução do plano de ação durante uma seqüência de tentativas organizadas de forma aleatorizada. A prática sob alta interferência contextual, por essa perspectiva, leva o executante a esquecer a solução para uma dada habilidade motora, de maneira que a solução deve ser gerada quando a tarefa é apresentada novamente. Em condição de baixa interferência contextual, por outro lado, o executante apenas manteria as especificações do plano de ação da tentativa anterior, sem precisar elaborar novos movimentos ao longo do processo de prática. Tudo que os aprendizes precisam fazer é relembrar e executar um único plano, a não ser na transição entre séries de tentativas. O processo repetido de reconstrução do plano de ação, por essa perspectiva, levaria à melhor retenção e transferência observadas na prática sob alta interferência contextual.

Desde a pesquisa seminal de Shea e Morgan, entretanto, foi estabelecida somente a fronteira das condições do efeito da interferência contextual. Hoje as pesquisam em interferência contextual tendem a se preocupar com os possíveis efeitos diferenciais da complexidade da tarefa praticada sobre o aprendiz. Desde a publicação da revisão de literatura de MAGILL e HALL (1990) têm sido apresentados resultados de pesquisas conflitantes com a hipótese da interferência contextual. Algumas possibilidades de explicação residem em aspectos tais como idade dos participantes, nível de habilidade, quantidade de prática e quantidade de interferência contextual. Sobre este ponto, OLLIS et al. (2005) destacaram a necessidade de se entender as relações entre experiência prévia e a dificuldade da tarefa com o efeito da interferência contextual para se obter melhores resultados de aprendizagem.

De um modo geral, devemos considerar como dificuldade da tarefa a necessidade de se ajustar ininterruptamente, e finalmente à capacidade de processamento das demandas 
colocadas na memória do aprendiz (cf. FLEISHMAN \& QUAINTANCE, 1984; GENTILE, 1998). De acordo GUADAGNOLI e LEE (2004), a aprendizagem ocorre em função da informação disponível e da interpretação do contexto do desempenho. O ponto ideal de aprendizagem é determinado por uma quantidade ótima de informação, que varia em função da habilidade do indivíduo que desempenha e da dificuldade da tarefa a ser aprendida. Assim, por este ponto de vista, entende-se que os efeitos pouco significantes da interferência contextual em determinadas condições experimentais podem ser resultado da elevada dificuldade nominal e funcional exigida no desempenho da tarefa, que não permite ao indivíduo desenvolver um ótimo ponto de aprendizagem. A dificuldade nominal reflete a quantidade fixa de dificuldade com respeito ao que é desempenhado (a tarefa) ou em que condição a tarefa é desempenhada. A dificuldade nominal está relacionada à interpretação do que vai ser desempenhado (informação disponível) e a demanda de controle motor. Quando a dificuldade nominal da tarefa é constante, a dificuldade funcional é relativa ao nível de habilidade de quem desempenha a tarefa. Por exemplo, ao se chutar uma bola de futebol em direção a um gol, o movimento do chute é caracterizado como dificuldade nominal, não muda. Porém, a dificuldade funcional (acertar o gol) é relativa à habilidade de quem desempenha o chute. Os autores concluem que, independentemente do nível de habilidade de um indivíduo, se analisáramos a dificuldade nominal de uma tarefa e a experiência de quem irá desempenhála o resultado será previsível. Para o iniciante, é esperado um pobre desempenho comparado a indivíduos mais experientes, ou a tarefa é tão fácil que qualquer um pode desempenhá-la com um nível alto de sucesso. Conforme a tarefa se torna mais difícil, é esperado que o nível de desempenho do indivíduo (iniciante intermediário ou experiente) caia rapidamente. Parindo desse princípio, de acordo com a proposta formulada por Guadagnoli e Lee, gerar uma quantidade adequada de informação no desempenho de uma tarefa, juntamente com o aumento da dificuldade funcional da tarefa, dificultando o desempenho da aquisição, paradoxalmente melhora a aprendizagem.

A aprendizagem é inicialmente relacionada à informação disponível. Por outro lado, ela depende da dificuldade funcional da tarefa. A informação é vista como um desafio para o aprendiz quando esta está potencialmente disponível. Alguns princípios derivados desta proposição são os seguintes: (a) a aprendizagem não pode ocorrer na ausência de informação, (b) a aprendizagem é retardada na presença informação demasiada ou de pouca informação, e (c) para ocorrer aprendizagem há necessidade de uma quantidade ótima de informação, que 
varia de acordo com o nível de habilidade do indivíduo e a dificuldade do que vai ser aprendido. GUADAGNOLI e LEE (2004), assim, ofereceram uma interpretação passível de teste empírico da interação da dificuldade da tarefa com o efeito da interferência contextual.

A partir das idéias apresentadas por GUADAGNOLI e LEE (2004), uma avaliação interessante seria com crianças. Uma razão fundamental desta proposta de investigação está relacionada às diferenças na capacidade de processamento de informações entre crianças e adultos o que pode ser importante para o efeito de interferência contextual. Pesquisadores têm mostrado que crianças têm capacidade reduzida de memória de trabalho quando comparado a adultos (KEOGH \& SUGDEN, 1985). Dessa forma, crianças não poderiam ser capazes de efetuar comparações com eficiência ou obter os mesmos benefícios de adultos. Na verdade, o número limitado de estudos em que crianças participaram gerou resultados diversos (EDNARDS, ELLIOT \& LEE, 1986; POLLOCK \& LEE, 1997). Apesar de PIGOTT e SHAPIRO (1984) não terem encontrado diferenças entre alta e baixa interferência contextual entre os grupos de adultos e crianças, no experimento de DEL REY, WHITEHURST, e WOOD (1983) foi encontrado um efeito inverso, com o grupo de prática em bloco apresentando desempenho superior na transferência quando comparado ao grupo de prática aleatória. No entanto, em um estudo de WEGMAN (1999) foi observado que os resultados dependeram do tipo de habilidade que foi praticada, com resultados distintos entre habilidades abertas e habilidades fechadas. Dado esses resultados diversos e a quantidade limitada de experimentos em que houve a participação de crianças, parece apropriado dar continuidade a pesquisas sobre a interferência contextual com esta faixa etária. Este ponto de vista tem sido compartilhado com diferentes pesquisadores (LANDIN \& HEBERT, 1997; MAGILL, 2001; NEWELL \& MCDONALD, 1992).

Considerando-se que o efeito da interferência contextual tem sido conceituado como independente do sistema muscular empregado durante a prática, uma importante lacuna identificada em estudos prévios é a carência de avaliação no que diz respeito à transferência interlateral de aprendizagem. Transferência interlateral de aprendizagem corresponde ao efeito da prática com um segmento corporal sobre o desempenho ou aprendizagem do membro contra lateral homólogo (MAGILL, 2001). O tema transferência interlateral passou a ser estudado por COOK (1933a, b) sob denominação de educação cruzada. A partir de antão, os achados vão de transferência interlateral nula (IOFFE, MASSION, GANTCHEV, DUFOSSÉ \& KULIKOV, 1996), assimétrica (TAYLOR \& HEILMAN, 1980; TEIXEIRA, 2000, 
Experimento 2) ou perfeitamente simétrica (IMAMIZU \& SHIMOJO, 1995, 1998). Particularmente no tocante aos resultados com o reduzido efeito de transferência, a prática com baixa interferência contextual talvez pudesse induzir resultados distintos.

Diferentes modelos conceituais têm sido propostos para explicar a transferência interlateral de aprendizagem, a saber: modelo de proficiência (LASZLO, BAGULEY \& BAIRTOW, 1970), modelo acesso calosal (TAYLOR \& HEILMAN, 1980), modelo de ativação cruzada (PARLOW \& KINSBOURNE, 1989), modelo de transferência visuomotora (IMAMIZU \& SHIMOJO, 1995). No modelo de proficiência Laszlo, é concebido que a transferência interlateral de aprendizagem se manifesta após a prática, pela irradiação dos benefícios de aprendizagem específica ao sistema neuromotor empregado através das comissuras cerebrais. Quando a análise da execução da ação é espacial, deve-se esperar transferência simétrica e mais eficiente principalmente em indivíduos destros, pois a aprendizagem se dará através da transferência interlateral da aprendizagem realizada com um dos membros do lado direito para o hemisfério cerebral esquerdo, pois o hemisfério cerebral esquerdo e membro direito é mais proficiente nos destros. O modelo de acesso calosal (TAYLOR \& HEILMAN, 1980) explica a transferência interlateral como um processo assimétrico, pois em destros os engramas seriam armazenados no hemisfério cerebral esquerdo, independentemente da mão usada na prática. O desempenho com a mão direita seria influenciado pela aprendizagem prévia da mão direita e da esquerda, a assimetria na transferência interlateral da aprendizagem para a mão esquerda é explicada pela necessidade de acesso inter-hemisférico através do corpo caloso gerando uma degradação da habilidade. Por este modelo se espera sempre encontrar pobre transferência de aprendizagem para o lado esquerdo. O modelo de ativação cruzada (PARLOW \& KINSBOURNE, 1989) propõe a formação de representações mentais das tarefas desempenhadas nos dois hemisférios cerebrais (engramas duplos) formando um sistema de ativação mais eficiente. Quando uma tarefa é desempenhada com um dos membros do lado direito, forma-se uma representação (engrama) no hemisfério cerebral esquerdo e um engrama paralelo no hemisfério direito. Porém, quando um indivíduo destro aprende uma tarefa com a mão esquerda, o engrama seria restrito ao hemisfério direito. Assim a prática com o lado contralateral ao hemisfério dominante promove a transferência interlateral de aprendizagem, enquanto que na prática com o sistema não dominante os efeitos são específicos a este sistema e não transferíveis. Este modelo é semelhante ao modelo de LASZLO, et al. (1970) com exceção a proposição de engramas 
duplos. O modelo de transferência visuomotora (IMAMIZU \& SHIMOJO, 1995) propõe que durante a aprendizagem de uma tarefa, há uma transformação das coordenadas espaciais percebidas pelo sistema visual, em comandos para o sistema muscular. Esta transformação ocorre hipoteticamente, em dois níveis na hierarquia de controle do movimento. O nível mais elevado (tarefa) é responsável por perceber as coordenadas espaciais externas do evento como os alvos ou objetos de trabalho, planejando as trajetórias dos segmentos corporais que interagirão com as coordenadas selecionadas. O nível mais baixo (manipulação) é constituído pela dinâmica inversa, os parâmetros cinemáticos devem ser transformados em torques articulares para produzir uma escala de respostas motoras apropriadas. A principal implicação desta conceituação é que se a aprendizagem realmente acontece em um nível mais elevado, uma forte transferência interlateral será esperada. Se a aprendizagem motora acontece em um baixo nível na hierarquia, ocorrerá uma aquisição efetora específica da tarefa como resultado da prática motora e fraca transferência interlateral de aprendizagem. Este modelo difere dos três anteriores por propor que não há qualquer assimetria de aprendizagem no processo.

Uma noção transversal aos modelos apresentados acima é que a melhor aprendizagem com o membro empregado durante as tentativas de aquisição deveria induzir uma transferência interlateral mais potente. Dessa forma, há base racional para hipotetizar que em havendo beneficio de aprendizagem ipsilateral ao membro usado na prática devido à interferência contextual, beneficio similar deveria ser observado em um teste de transferência interlateral de aprendizagem.

\section{OBJETIVO}

O objetivo deste estudo foi verificar o efeito do contexto de variação da prática - em blocos versus aleatória - sobre a transferência ipsilateral e contralateral de padrões gráficos em crianças.

À medida que existem muitas controvérsias na literatura com relação ao efeito da interferência contextual aplicado a crianças, neste estudo comparamos se a prática em bloco 
leva vantagem sobre a prática aleatória na aprendizagem de padrões gráficos e resulta em melhora na retenção e transferência (ipsilateral e contralateral) da habilidade.

\section{MÉTODO}

\subsection{Participantes}

Participaram do estudo, 30 crianças destras matriculadas na Escola Estadual Prof. Joaquim Nabuco para o ano letivo de 2008. Foram selecionadas crianças de ambos os sexos, 15 meninos e 15 meninas, com idade cronológica de 8-9 anos. A mão preferida direita foi estabelecida com base na mão usada para escrever. Foi colocado um lápis sobre uma folha de papel e solicitou-se ao indivíduo que pegasse o lápis e escrevesse seu nome. Através da observação do experimentador verificou-se qual mão foi utilizada para escrever o nome, certificando-se que o indivíduo era destro. Posteriormente a esta pré-seleção a admissão como participante foi feita mediante o retorno do consentimento informado obtido de cada criança e do respectivo responsável (ANEXO 1).

\subsection{Tarefa e equipamentos}

A tarefa consistiu em contornar figuras geométricas visualizando-as em um monitor de computador. As figuras empregadas no estudo foram as seguintes: círculo, cruz, triângulo eqüilátero e diamante (FIGURA 1). O espaço de traçado nas figuras geométricas foi delimitado pela espessura da linha da figura exibida na tela do monitor do computador, correspondendo a 1,5 cm. Foi utilizado um computador portátil (monitor de 14 polegadas), onde, por meio de um programa computacional desenvolvido para este estudo, a tarefa foi realizada. O participante desempenhou o traçado da figura sobre uma mesa digitalizadora usando uma caneta eletrônica. As figuras tinham a dimensão de: círculo $(9,7 \mathrm{~cm}$ comprimento x 9,7 cm largura); cruz ( $8 \mathrm{~cm}$ comprimento x $8 \mathrm{~cm}$ largura); triângulo (10,7 cm comprimento x 10,3 cm largura); diamante (11,6 cm comprimento x $14 \mathrm{~cm}$ largura). A mesa digitalizadora foi disposta em posição invertida, fazendo com que o traçado na tela fosse o inverso daquele realmente feito pelo participante. Isto é, movimentos feitos para a direita produziram deslocamento do cursor na tela para esquerda, com a mesma inversão ocorrendo no eixo 
vertical. A tarefa dos participantes foi completar o traçado das figuras com o cursor na tela no menor período de tempo sem exceder os limites espaciais estabelecidos. Os participantes realizaram a tarefa sentados, tendo o monitor disposto em ângulo reto com a mesa à sua frente a aproximadamente $50 \mathrm{~cm}$ de distância.

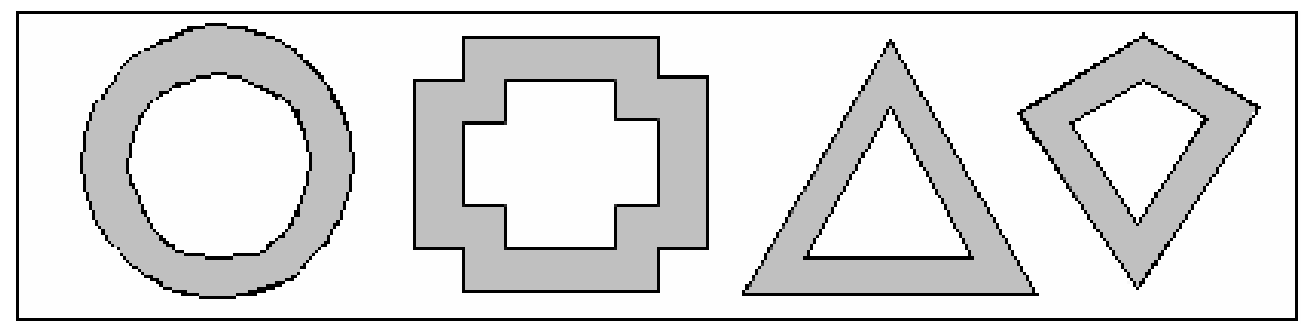

FIGURA 1 - Formas geométricas empregadas na fase de prática (círculo, cruz e triângulo eqüilátero) e na fase de transferência (diamante).

\subsection{Delineamento experimental e procedimentos}

As crianças foram designadas aleatoriamente a três grupos, havendo 10 participantes (cinco meninas e cinco meninos) em cada um: grupo alta interferência contextual (AL), programa de prática de contorno das três figuras geométricas com seqüência aleatorizada entre as figuras; grupo baixa interferência contextual (BA), programa em que cada figura geométrica foi praticada em blocos de tentativas em cada sessão; e grupo controle (CO), que não praticaram as tarefas da fase de aquisição.

O experimento teve quatro fases: aquisição, retenção, transferência ipsilateral, e transferência contralateral. Preliminarmente a todas as primeiras sessões da fase de aquisição, todo o aparato era montado em uma sala isolada das demais dependências da escola e posteriormente o experimentador dirigia-se ao local onde o indivíduo se encontrava (sala da aula) e acompanhava-o ao local do experimento. Este procedimento foi adotado somente para os primeiros indivíduos a cada sessão do experimento e logo após o recreio, os demais indivíduos eram chamados pelo anterior ao término de sua sessão de tentativas.

$\mathrm{Na}$ chegada das crianças ao local do experimento, previamente ao início do desempenho das tarefas, elas eram orientadas a sentar confortavelmente na cadeira de forma que suas costas estivessem em contato com o encosto da cadeira e seus pés estivessem em contato com o solo. Em seguida o aparato era apresentado ao indivíduo (computador, mesa 
digitalizadora e a caneta digital). Antes que o indivíduo entrasse em contato com o aparato, era-lhe apresentado um mouse que se encontrava sobre a mesa e não estava conectado ao computador. Explicava-se que aquele mouse seria substituído pela caneta digital para que ele desempenhasse as tarefas. Logo após, mostrava-se o cursor na tela do computador e o experimentador deslocava-o com a caneta digital.

Após finalizado os procedimentos iniciais tinha início a tentativa de familiarização que foi cronometrada pelo experimentador por meio de um cronômetro manual. O experimentador inicialmente deixava a criança deslocar a caneta digital livremente durante $30 \mathrm{~s}$. Em seguida, solicitava-se à criança que parasse e perguntava-se o que ela havia percebido ao observar o deslocamento do cursor. A partir de então, através da orientação do experimentador era solicitado que a criança deslocasse o cursor a pontos pré-determinados na tela do computador (arrastar o cursor para o ângulo superior direito, para o ângulo inferior esquerdo; para a região central; para cima ao centro, para baixo ao centro; para a região central; para a região central esquerda, para a região central direita) com duração de 90 s. Em seguida pedia-se a criança que parasse o desempenho da tarefa de familiarização e apresentava-se o botão que deveria ser pressionado na caneta digital para que o cronômetro do programa pudesse ser iniciado. Para que a criança pudesse vivenciar e sentir como pressionar o botão da caneta e qual pressão deveria ser exercida, o experimentador pedia que a criança pressionasse algumas vezes o botão, porém à distância da mesa digitalizadora de forma que não houvesse efeito de aprendizagem. A partir de então tiveram início as tentativas da fase de aquisição.

$\mathrm{Na}$ fase de aquisição os participantes dos grupos experimentais (AL e BA) receberam 180 tentativas de prática distribuídas em quatro semanas (ANEXO 2). A cada dia de prática os grupos realizavam uma sessão de 15 tentativas obedecendo à respectiva programação de prática. As tentativas foram realizadas com a mão direita, com o contorno das figuras sendo iniciado na parte inferior central da figura e os movimentos feitos no sentido horário. Caso o participante excedesse os limites do traçado das figuras ele retornava ao mesmo ponto da figura onde excedeu a linha divisória e dava continuidade ao contorno da figura. Em cada tentativa eram permitidos dois erros no máximo. Caso contrário, a tentativa era reiniciada. Ao final de cada tentativa era informado ao participante sobre o tempo de movimento daquela tentativa.

Um dia após a última sessão de prática, todos os participantes realizaram o teste de retenção. Esse teste foi composto de três tentativas da figura cruz, com o mesmo propósito da 
sessão de prática. Isto é, completar o traçado no menor período de tempo. Imediatamente após o teste de retenção, era realizado o teste de transferência ipsilateral. Esse teste foi compreendido por três tentativas no contorno da figura diamante, sendo o traçado da figura feito no mesmo sentido (horário) das demais figuras. Essa figura foi selecionada pelo fato de possuir quatro linhas obliquas que exigem movimentos que não foram feitos nas tentativas de prática. Em seguida era feita a avaliação de transferência contralateral, realizando-se três tentativas da tarefa de transferência com a mão contralateral àquela empregada para a prática (esquerda), fazendo-se os movimentos no sentido anti-horário.

\subsection{Análise dos dados}

A partir dos dados de tentativas individuais foram calculados o tempo de movimento médio e a variabilidade de tempo de movimento (para 3 tentativas), dada pelo cálculo do desvio padrão. A fase de aquisição foi subdividida em blocos de três tentativas para cada figura. O modelo estatístico foi de análise de variância (ANOVA) de três fatores 2 (grupo BA x AL) x 3 (figura) x 5 (bloco $[1,5,10,15,20]$ ), com medidas repetidas nos dois últimos fatores. Os resultados das fases principais foram avaliados por meio de ANOVAs de dois fatores, 3 (grupo) x 3 (fase: retenção transferência ipsilateral x transferência contralateral), com medidas repetidas no segundo fator. Comparações post hoc foram feitas por meio do teste Newman-Keuls. Em todas as análises o nível mínimo de significância foi estabelecido em 5\%.

$6 \quad$ RESULTADOS $^{1}$

\subsection{Tempo de Movimento}

\subsubsection{Fase de aquisição}

A análise do TM na fase de aquisição mostrou diferenças significantes no fator principal bloco $[\mathrm{F}(4,72)=39,38, \mathrm{p}<0,01]$, que foi devida a valores mais elevados de TM apenas no primeiro bloco de tentativas em relação aos demais. Não foram encontrados efeitos

\footnotetext{
${ }^{1}$ Ver tabelas das análises de variância no Anexo
} 
significantes para o fator principal grupo nem para interação entre os fatores principais (FIGURA 2).

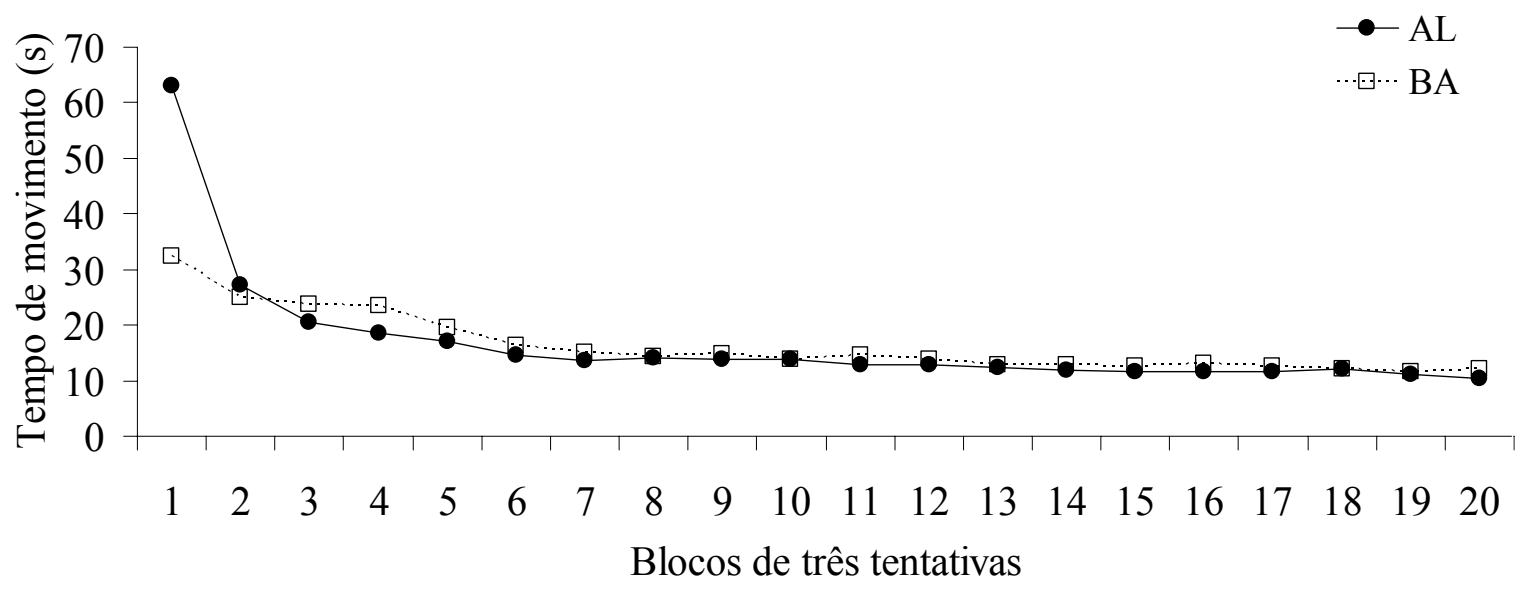

FIGURA 2 - Média de tempo de movimento para blocos de três tentativas durante a fase de aquisição dos grupos AL e BA.

\subsubsection{Retenção e transferência}

$\mathrm{Na}$ fase de retenção a análise estatística mostrou diferença significante nos fatores principais grupo $[\mathrm{F}(2,27)=25,31, \mathrm{p}<0,01]$ e teste $[\mathrm{F}(2,54)=14,72, \mathrm{p}<0,01]$. As comparações post hoc indicaram que o efeito de grupo foi devido a TMs mais altos no grupo controle em comparação aos grupos experimentais, que não diferiram entre si. O efeito de teste foi devido TMs mais elevados na transferência contralateral em comparação à retenção e transferência ipsilateral, que não diferiram entre si (FIGURA 3). 


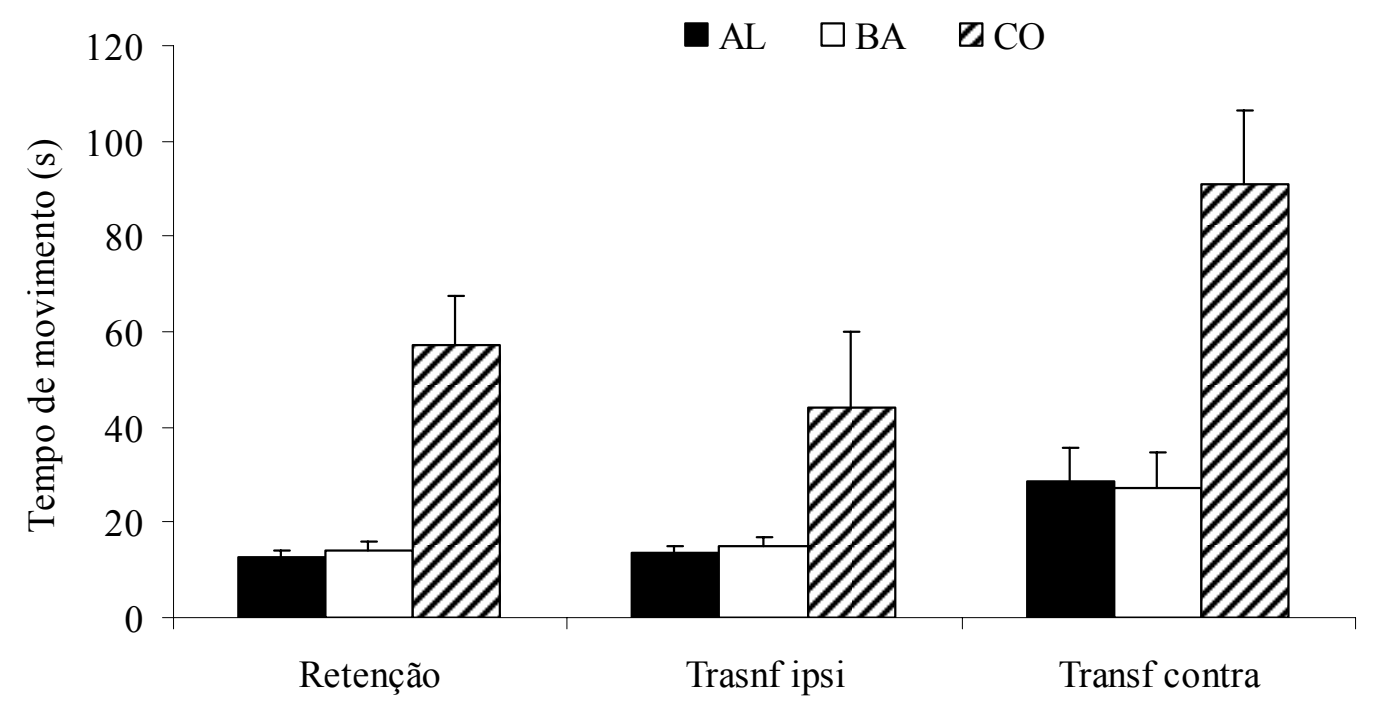

FIGURA 3 - Média de TM para os grupos experimentais (AL, BA) e controle (CO) em função do teste: retenção, transferência ipsilateral e transferência contralateral.

6.2 Variabilidade de tempo de movimento

\subsubsection{Fase de aquisição}

A análise de variabilidade do TM na fase de aquisição mostrou diferença significante apenas no fator principal bloco $[\mathrm{F}(4,72)=13,45, \mathrm{p}<0,01]$. As comparações post hoc indicaram que a variabilidade do TM foi mais elevada no primeiro bloco de tentativas em relação aos demais blocos, que não diferiram entre si (FIGURA 4).

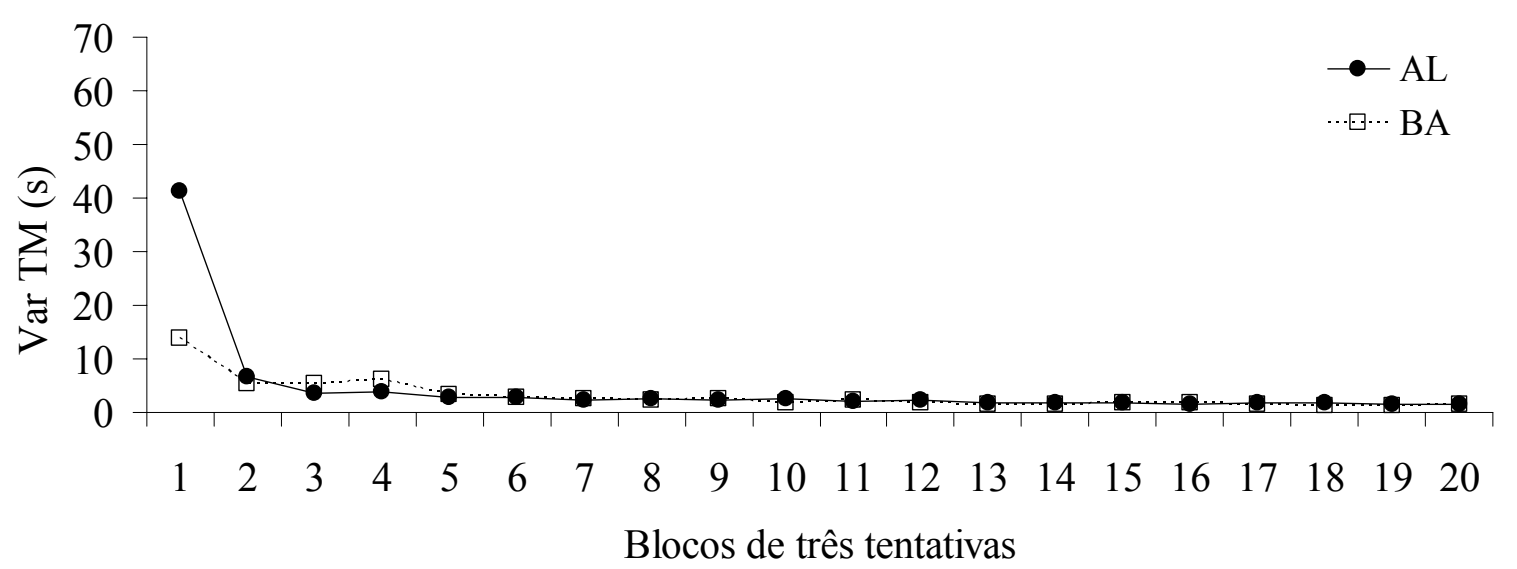

FIGURA 4 - Média da variabilidade do tempo de movimento a cada três tentativas no contorno das figuras geométricas durante a fase aquisição para os grupos AL e BA. 


\subsection{Retenção e transferência}

A análise de variabilidade do TM mostrou efeitos significantes dos fatores principais grupo $[F(2,27)=35,13, p<0,01]$ e teste $[F(2,54)=14,37, p<0,01]$. As comparações post hoc indicaram que o efeito de grupo foi devido à variabilidade de TM mais elevada no grupo controle em comparação aos grupos experimentais, que não diferiram entre si. $\mathrm{O}$ efeito de teste foi devido à variabilidade de TM mais elevada na transferência contralateral em comparação a retenção e transferência ipsilateral, que não diferiram entre si (FIGURA 5).

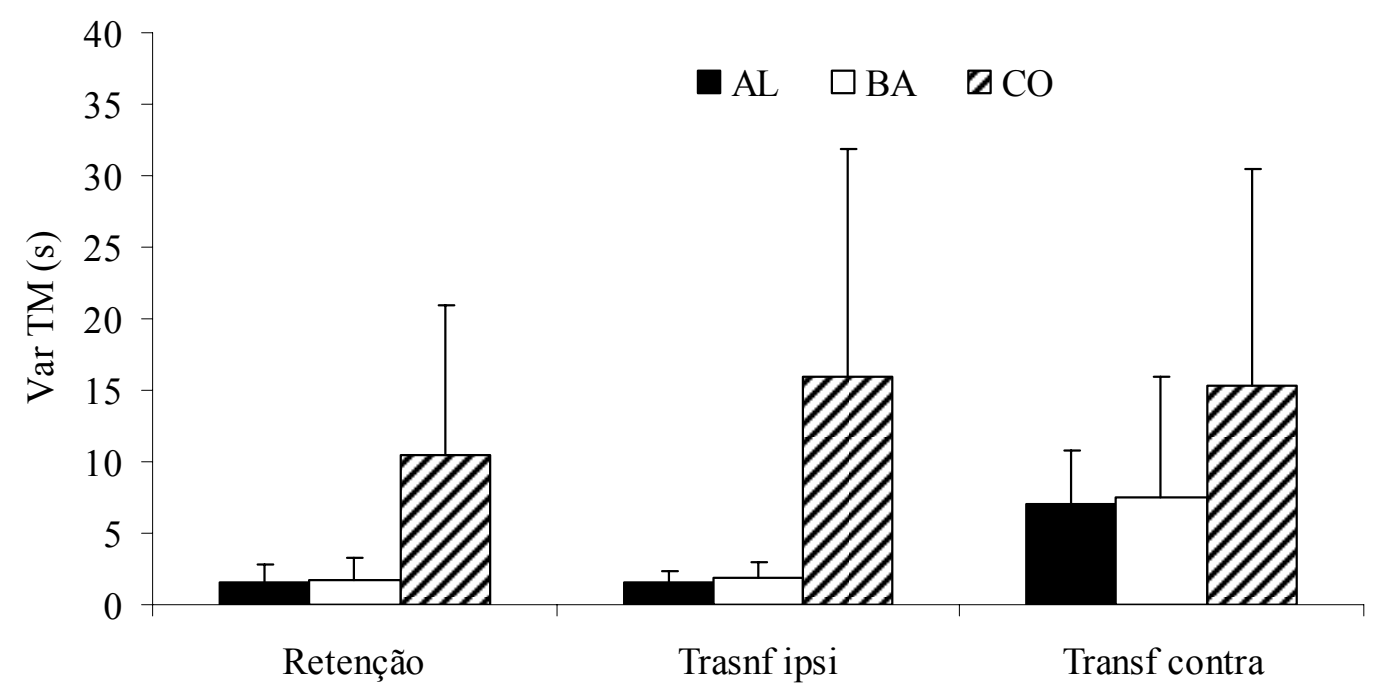

FIGURA 5 - Média da variabilidade de TM para os grupos experimentais (AL, BA) e controle $(\mathrm{CO})$ em função do teste: retenção, transferência ipsilateral e transferência contralateral.

No presente estudo foi testada a hipótese de que a alta dificuldade funcional de uma tarefa reverteria o efeito de interferência contextual (IC), fazendo com que baixa IC levasse a efeitos mais positivos de aprendizagem do que alta IC em situações de retenção e transferência (cf. GUADANOLI \& LEE, 2004). Para conduzir este teste, foram comparados os efeitos de IC em crianças, em fase de aprendizagem da escrita, na aquisição, transferência ipsilateral e transferência contralateral de padrões gráficos com feedback visual invertido. Com este arranjo experimental buscou-se criar condições de alta dificuldade funcional durante a prática 
da tarefa, avaliando-se a transferência não apenas para o sistema muscular empregado durante a prática na tarefa, mas também para o membro que permaneceu em repouso. Esse último aspecto conferiu uma avaliação mais ampla dos efeitos de transferência em relação aos delineamentos experimentais previamente empregados na investigação da IC na aprendizagem de ações motoras.

Os resultados mostraram que durante a prática dos padrões gráficos foi observada uma diferença significante entre os grupos experimentais apenas no primeiro bloco de tentativas, com progressivo ganho de desempenho na tarefa de forma similar entre os dois grupos. Nas fases de retenção e transferência encontrou-se uma vantagem consistente dos grupos experimentais em relação ao grupo controle, porém nenhuma diferença significante foi detectada entre os grupos experimentais. De forma original neste trabalho, foi evidenciado que a adaptabilidade adquirida pela prática variada dos padrões gráficos tanto em baixa quanto em alta IC foi transferida não apenas para o braço ativo durante a prática, mas também para o braço contralateral.

A despeito de não ter sido observado o efeito hipotetizado de melhor retenção e transferência com baixa IC em uma tarefa considerada de alta dificuldade funcional, os presentes resultados mostram que a aleatorização da prática não induziu efeitos mais positivos de aprendizagem como resultado de alta IC. Este resultado é reminiscente de achados mostrando que na aprendizagem de habilidades gráficas com diferentes graus de dificuldade nominal em adultos a vantagem da alta IC é observada nas tarefas mais simples, mas não em tarefas mais complexas (ALBARET \& THON, 1998). Além disso, DEL REY et al. (1982) mostraram que na comparação entre indivíduos habilidosos versus novatos em habilidades motoras abertas, a prática de uma tarefa requisitando antecipação de coincidência leva a efeitos diversos. Nesta situação, a vantagem da aleatorização da prática foi observada apenas em participantes habilidosos, enquanto que nos novatos o melhor resultado de aprendizagem foi atingido com mínima variação da prática (ver também GUADAGNOLI et al., 1999; HEBERT et al., 1996). Desta forma, aparentemente a alta dificuldade funcional de uma tarefa parece atenuar, ou em certos casos reverter, a vantagem da alta IC na aprendizagem de habilidades motoras.

Uma possível explicação para os efeitos menos evidentes da dificuldade nominal da tarefa, induzindo os mesmos resultados tanto em baixa quanto em alta IC, é a quantidade de prática relativamente extensa distribuída por diferentes dias. Nesta situação há potencialização 
dos efeitos de prática pela consolidação das modificações na memória de longa duração (WALKER et al., 2003), o que pode ter contribuído para uma melhora precoce do grupo praticando com alta IC. Como observado nos resultados, o grupo AL teve um desempenho expressivamente inferior ao grupo BA apenas no primeiro bloco de tentativas. Nos demais blocos o desempenho entre os grupos foi bastante semelhante. A partir desta observação, fica aparente que a dificuldade funcional da tarefa para o grupo AL foi reduzida precocemente durante a prática, tornando o desafio de executar a tarefa rapidamente similar entre os dois grupos experimentais. Esta conclusão é consistente com os resultados de SHEA, KOHL e INDERMILL (1990) mostrando que o benefício da prática sob alta IC se manifesta após muitas tentativas de prática, mas não quando a prática é limitada a um número reduzido de tentativas. A partir deste resultado, é hipotetizado que a baixa IC poderia beneficiar a retenção e transferência durante as fases iniciais, enquanto que em fases mais avançadas a alta IC seria mais benéfica. A semelhança dos efeitos de aprendizagem entre os dois grupos experimentais no presente estudo, assim, poderia ser devida à interação entre IC e fase de aprendizagem.

Uma hipótese alternativa, porém não exclusiva, é de que a alta plasticidade neural característica de crianças nesta idade (HAYWOOD \& GETCHELL, 2004) poderia também modular os efeitos de IC. Como observado usualmente em estudos com adultos, durante a fase de prática alta IC induz desempenhos inferiores durante a prática e efeitos superiores durante os testes de retenção e transferência (cf. MAGILL \& HALL, 1990). Nesse sentido, a rápida adaptação à alta IC precocemente durante a prática nas crianças poderia ser devida à maior plasticidade neural. O resultado de tal adaptação em fases iniciais de aquisição da habilidade poderia tornar a aprendizagem insensível ao efeito de IC. A partir desta noção, os dados sugerem também que o prejuízo de desempenho durante a prática é um elemento importante para a obtenção de efeito positivo sobre a retenção e transferência de aprendizagem.

A análise dos resultados de transferência intermanual de aprendizagem mostraram uma vantagem de desempenho dos grupos experimentais sobre o grupo controle. Este dado mostra originalmente a generalização intermembros da capacidade de controle motor nos padrões gráficos praticados para um padrão distinto, requisitando traços diferentes e em sentido contrário àquele praticado. Tais resultados estendem a noção de aprendizagem em um nível abstrato de representação, em que nem o sistema muscular ou movimentos específicos são codificados na memória motora (cf. IMAMIZU \& SHIMOJO, 1995, 1998; TEIXEIRA, 2006). Em outras palavras, uma parte dos ganhos devidos à prática dos padrões gráficos poderia ser 
desenvolvida simultaneamente nos dois hemisférios cerebrais por meio do acesso calosal ou, alternativamente, em uma região de acesso comum entre os dois hemisférios cerebrais. $\mathrm{O}$ declínio de desempenho na comparação entre as tarefas de transferência ipsi e contralateral, por outro lado, revela que parte da aprendizagem foi específica à tarefa. Esse achado indica a aprendizagem como resultado de diferentes níveis de codificação na memória (cf. TEIXEIRA \& CAMINHA, 2003). Uma vez que não foi encontrado efeito de IC, entretanto, os resultados aqui relatados não permitem uma conclusão inequívoca sobre uma transferência intermanual diferenciada devida à programação da variabilidade de prática durante a aquisição de uma habilidade motora.

8

\section{CONCLUSÕES}

Os resultados do presente estudo conduziram às seguintes conclusões:

1. Não houve efeito de IC em nenhuma das fases do experimento, refutando a hipótese de que baixa IC nas condições avaliadas levaria aos melhores resultados nas etapas de retenção e transferência.

2. A prática variada induziu efeitos significantes de transferência ipsilateral e de transferência contralateral, estendo os achados sobre adaptabilidade devida à IC.

9 LIMITAÇÕES DO TRABALHO

A falta de comparação com a aprendizagem de uma tarefa motora mais simples dificulta uma avaliação rigorosa da hipótese formulada por GUADAGNOLI e LEE (2004), uma vez que não se possui informação sobre o efeito de IC na população estudada em uma tarefa de dificuldade funcional mais baixa.

A carência de bons parâmetros cinemáticos para uma avaliação mais refinada dos movimentos levou à limitação da análise ao tempo total de movimento. 
A partir da interpretação dos resultados apresentada na discussão e das limitações apresentadas na seção anterior, sugere-se a comparação entre tarefas mais simples e mais complexas na avaliação da proposta de efeito diferencial de IC em função da dificuldade funcional da tarefa. Neste sentido, algo importante em tal avaliação seria uma análise preliminar da efetiva dificuldade da tarefa. Isso poderia ser efeito tanto pelo desempenho motor quanto pela atenção requisitada na execução da tarefa.

Em uma análise geral da literatura, percebe-se que o estudo dos aspectos diferenciais da aprendizagem motora em crianças tem sido relegado a segundo plano. A partir desta observação, sugere-se que em futuros estudos sejam incluídos grupos de comparação compostos por adultos. Tal comparação entre resultados de adultos e crianças poderia indicar diferenças importantes da capacidade de generalização entre tarefas motoras devida ao efeito de IC. 


\section{REFERÊNCIAS BIBLIOGRÁFICAS}

ALBERET, J.M; THON, B. Differential effects of task complexity on contextual interference in a drawing task. Acta Psychologica, v.100, n.1, p.9-24, 1998.

BABO, A.G.F.; AZEVEDO NETO, R.M.; TEIXEIRA, L. A. Adaptability from contextual interference in the learning of an open skill is context dependent. The Open Sports Medicine Journal, v.2, p.56-9, 2008.

BATTIG, W.F. Facilitation and interference. In: BILODEAU, E.A. Acquisition of skill. New York: Academic Press, 1966, p.215-44.

BRADY, F. Contextual interference: a meta-analityc study. Perceptual and Motor Skills, v.99, p.116-26, 2004.

COOK, T.W. Studies in cross education: I. Mirror tracing the star-shaped maze. Journal of Experimental Psychology, v.16, p.144-60, 1933a.

COOK, T.W. Studies in cross education: II Further experiments in mirror tracing the starchaped maze. Journal of Experimental Psychology, v.16, p.679-700, 1933 b.

DEL REY P.; WUGHALTER E.H.; WHITEHURST, M. The effects of contextual interference on females with varied experience in open sport skills. Research Quarterly for Exercise and Sport, v.53, p.108-15, 1982b.

DEL REY, P.; WHITEHURST, M.; WOOD, J. Effects of experience and contextual interference on learning and transfer. Perceptual Motor Skills, v.546, p.581-2, 1983.

EDWARDS, J.M., ELLIOT, D.; LEE, T.D. Contextual interference effects during skill acquisition and transfer in Down's syndrome adolescents. Adapted Physical Activity Quarterly, v.3, p. 250-8, 1986. 
FLEISHMAN, E.A.; QUAINTANCE, M.K. Taxonomies of human performance: The description of human tasks. Orlando: Academic Press, 1984.

GENTILE, A.M. Implicit and explicit processes during acquisition of functional skills.

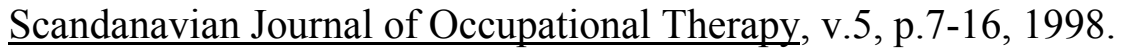

GUADAGNOLI, M.A.; HOLCOMB, W.R.; WEBER T.J. The relationship between contextual interference effects and performer expertise of the learning of a putting task. Journal of Human Movement Studies, v.37, n.1, p.19-36, 1999.

GUADAGNOLI, M.A.; LEE, T.D. Challenge point: a framework for conceptualizing the effects of various practice conditions in motor learning. Journal of Motor Behavior, v.36, n.2, p.212-3, 2004.

HAYWOOD, K.M.; GETCHELL, N. Desenvolvimento motor ao longo da vida. Porto Alegre: Artmed, 2004.

HEBERT, E.P.; LANDIN, D.; SOLMON, M.A. Practice schedule effects on the performance and learning of low- and high-skilled students: an applied study. Research Quarterly for Exercise and Sport, v.67, p.52-8, 1996.

IMAMIZU, H.; SHIMOJO, S. The lócus of visual-motor learning at the task or manipulator level: Implications for intermanual transfer. Journal of Experimental Psychology: Human Perception and Performance, v.4, n.21, p.719-33, 1995.

IMAMIZU, H.; UNO, Y; KAWATO, M. Adaptive internal model of intrinsic kinematics involved in learning an aiming task. Journal of Experimental Psychology: Human Perception and Performance, v.24, n.3, p.812-9, 1998.

IOFFE, M.; MASSION, J.; GANTCHEV, N.; DUFOSSE, M.; KULIKOV, M.A. Coordination between posture and movement in a bimanual load-lifting task: is there a transfer. Experimental Brain Research, v.109, n.3, p.450-6, 1996. 
KEOGH, J.; SUGDEN, D. Movement skill development. New York: MacMillan, 1985.

LANDIN, D.; HEBERT, E.P. A comparison of three practice schedules along the contextual interference continuum. Research Quarterly for Exercise and Sport, v.68, p.357-61, 1997.

LASZLO, J.I.; BAGULEY, R.A.; BAIRSTOW, P.J. Bilateral transfer in tappin skill in the absense of peripheral information. Journal of Motor Behavior, v.2, p.267-71, 1970.

LEE, T.D.; MAGILL, R.A. Can forgetting facilitate skill acquisition? In: GOODMAN, D.; WILBERG, R.B.; FRANKS, I.M. Differing perspectives on memory, learning and control. Amsterdam: Elsevier North Holland, 1985, p.3-22.

LI, Y.; LIMA, R.P. Rehearsal of task variations and contextual interference effect a field setting. Perceptual and Motor Skills, v.94, p.750-2, 2002.

MAGILL, R.A.; HALL, K.G. A review of the contextual interference effect in motor skill acquisition. Human Movement Science, v.9, p.241-89, 1990.

MAGIL, R.A. Aprendizagem motora: Conceitos e aplicações. São Paulo: Edgard Blucher Ltda, 2001.

MASLOVAT, D.; CHUA, R.; LEE, T.D.; FRANKS, I.M. Contextual interference: single task versus multi-task learning. Motor control, v.8, p.213-33, 2004.

NEWELL, K.M.; McDONALD, E.V. Searching for solutions to the coordination function: Learning as exploratory behavior. In: STELMACH, G.E.; REQUIN, J. Tutorials in motor behavior 2. Advances in psychology. Amsterdam: North-Holland, 1992, v.87, p.517-32.

OLLIS, S.; BUTTON, C.; FAIRWATHER, M. The influence of professional expertise and task complexity upon the potency of the contextual interference effect. Acta psychological, v.118, p.229-44, 2005. 
PARLOW, S.E.; KINSBOURNE, M. Asymmetrical transfer of training between hands: Implications for interhemisferic communication in normal brain. Brain and Cognition, v.11, p.98-113, 1989.

PIGOTT, R.E.; SHAPIRO, D.C. Motor schema: the structure of the variability session. Recearch Quartely for Expertise and Sport, v.55, n.1, p.41-5, 1984.

POLLOCK, B.J.; LEE, T.D. Dissociated contextual interference effects in children and adults. Perceptual \& Motor Skills, v.84, p.851-8, 1997.

SCHMIDT, R.A. A schema theory of discrete motor skill learning. Psychological Review, v.82, n.4, p.225-60, 1975.

SHEA, J.B.; MORGAN, R.L. Contextual interference effects on the aquisition, retention, and transfer of a motor skill. Journal of Experimental Psychology: Human Learning and Memory, v.5, n.2, p.179-87, 1979.

SHEA, C.H.; WULF, G. Schema theory: a critical appraisal and reevaluation. Journal of Motor Behavior, v.37, n.2, p.85-102, 2005.

SHEA, J.B.; ZIMNY S.T. Context effects in memory and learning movement information. In: MAGILL, R.A. Memory and control of action. Amsterdam: North-Holland, 1983, p.345-66.

SHEA, C.H.; KOHL, R.; INDERMILL, C. Contextual interference: contributions of practive. Acta psychologica, v.73, n.2, p.145-57, 1990.

SMITH, P.J.K.; GREGORY, S.K.; DAVIES, M. Alternating versus blocked practice in learning a cartwheel. Perceptual and Motor Skills, v.96, n.2, p.1255-64, 2003.

STE-MARIE, D.M.; CLARK, S.E.; FINDLAY, L.C.; LATIMER, A.E. High levels of contextual interference enhance handwriting skill acquisition. Journal of Motor Behavior, v.36, n.1, p.112-5, 2004. 
TAYLOR, H.G; HEILMAN K.M. Left-hemisphere motor dominance in right-handers. Cortex, v.16, p.587-603, 1980.

TEIXEIRA, L.A.; CAMINHA, L.Q. Intermanual transfer of force control is modulated by muscular strength. Experimental Brain Research, v.149, p.312-9, 2003.

TEIXEIRA, L.A. Timing and force components in bilateral transfer of learning. Brain and Cognition, v.44, p.455-69, 2000.

TEIXEIRA, L.A. Intermanual transfer of timing control between tasks holding different levels of motor complexity. Laterality, v.11, n.1, p.43-56, 2006.

VAN ROSSUM, J.H.A. Schmidt's schema theory: the empirical base of the variability of practice hypoteses: a critical analysis. Human Movement Science, v.9, n.3-5, p.387-435, 1990.

VERA, G.V.; MONTILLA, M.M. Practice schedule and acquisition, retention and transfer of a throwing task in 6-yr.-old children. Perceptual and Motor Skills, v.96, p.1015-24, 2003.

WALKER, M.P.; BRAKEFIELD, T.; HOBSON, J.A.; STICKGOLD, R. Dissociable stages of human memory consolidation and reconsolidation. Nature, v.425, p.616-20, 2003.

WEGMAN, E. Contextual interference effects on the acquisition and retention of fundamental motor skills. Perceptual and Motor Skills, v.88, p.182-7, 1999.

WRIGHT D.L; BLACK C.B.; BRUCKNER S.; MAGNUSON, C. Long-term motor programming improvements occur via concatenation of movement sequences during random but not during blocked practice. Journal Motor Behavior, v.36, n.1, p.39-50, 2004.

WRIGHT, D.L.; MAGNUSON, C.E.; BLACK, C.B. Programming and reprograming sequence timing following high and low contextual interference practice. Research Quartely for Exercice and Sport, v.76, n.3, p.258-66, 2005. 
ANEXO I - Termo de consentimento livre e esclarecido

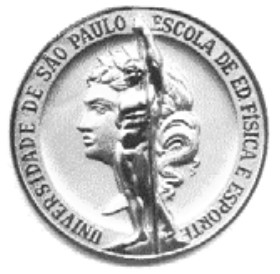

ESCOLA DE EDUCAÇÃO FÍSICA E ESPORTE DA UNIVERSIDADE DE SÃO PAULO - USP

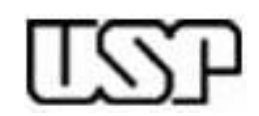

TERMO DE CONSENTIMENTO LIVRE E ESCLARECIDO

I - DADOS DE IDENTIFICAÇÃO DO SUJEITO DA PESQUISA OU RESPONSÁVEL LEGAL

1. NOME DO INDIVÍDUO:

DOCUMENTO DE IDENTIDADE: SEXO: $\mathrm{M} \square \mathrm{F}$

DATA DE NASCIMENTO (......... .........

ENDEREÇO $\mathrm{N}^{\mathrm{o}} \ldots . .$. APTO .....

BAIRRO:

CIDADE:

CEP: TELEFONO: DDD

$(\ldots \ldots .$.

2. RESPONSÁVEL LEGAL:

NATUREZA (grau de parentesco, tutor, curador, etc.):

DOCUMENTO DE IDENTIDADE: SEXO: $\mathrm{M} \square \mathrm{F} \square$

DATA DE NASCIMENTO

..................

ENDEREÇO: $\mathrm{N}^{\mathrm{O}} \ldots \ldots$.... APTO .....

BAIRRO:

CIDADE:

CEP: TELEFONO: DDD ).

\section{II - DADOS SOBRE A PESQUISA CIENTÍFICA}

1. TÍTULO: O Efeito da Interferência Contextual na Aprendizagem do Contorno de Figuras Geométricas e Transferência Inter-lateral do Movimento

2. PESQUISADOR RESPONSÁVEL: LUIS AUGUSTO TEIXEIRA

3. CARGO/FUNÇÃO: Professor associado no departamento de Biodinâmica da Escola de Educação Física e Esporte - USP

4. AVALIAÇÃO DO RISCO DA PASQUISA:

$\begin{array}{lll}\text { RISCO MÍNIMO } & 6 & \text { RISCO MÉDIO } \\ \text { RISCO BAIXO } & \square & \text { RISCO MAIOR }\end{array}$

(probabilidade de que o indivíduo sofra algum dano como conseqüência imediata ou tardia do estudo)

5. DURAÇÃO DA PESQUISA: Uma Semana; 40 minutos por dia.

\begin{tabular}{|c|}
\hline I \\
\hline
\end{tabular}
REPRESENTANTE LEGAL SOBRE A PESQUISA, CONSIGNANDO: 
1 - A presente pesquisa tem como finalidade investigar os efeitos de diferentes tipos de programas de prática (bloco, aleatório) na aprendizagem da habilidade no contorno de figuras geométricas e a conseqüente transferência inter-lateral do movimento.

2 - O sujeito realizará o contorno de figuras geométricas em uma mesa digitalizadora com uma caneta especial, seguindo figuras apresentadas na tela do computador.

3 - Não são esperados nenhum desconforto ou risco durante a coleta.

\section{IV - ESCLARECIMENTOS DADOS PELO PESQUISADOR SOBRE GARANTIAS DO SUJEITO DA PESQUISA:}

1 - Você terá disponível a qualquer momento e por qualquer motivo seus registros e resultados para serem consultados ou até mesmo retirados de acordo com a sua vontade. Seus resultados serão registros numéricos referentes ao seu desempenho (registrados digitalmente).

2 - Você terá liberdade de retirar seu consentimento ou deixar de participar do estudo a qualquer momento, sem que isto traga prejuízo ou qualquer transtorno.

3 - Os seus resultados obtidos durante o experimento poderão ser publicados. Porém, será assegurado à privacidade e sigilo absoluto sobre seus resultados, nome, imagens ou dados pessoais.

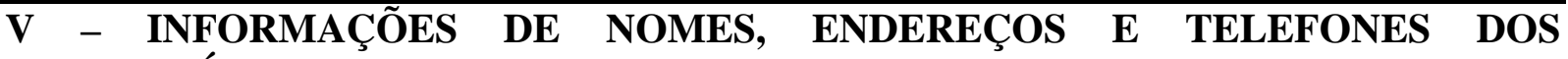 RESPONSÁVEIS PELO ACOMPANHAMENTO DA PESQUISA, PARA CONTATO EM CASO DE DÚVIDAS:}

Pesquisador: Alexandre Gomide Frugiuele Babo

Orientador: Prof. Dr. Luis Augusto Teixeira

Endereço: Escola de Educação Física e Esporte - USP

Telefone: 3091-2129

\section{VII - CONSENTIMENTO PÓS-ESCLARECIDO}

Declaro que, após convenientemente esclarecido pelo pesquisador e ter entendido o que me foi explicado, consinto em participar do presente Projeto de Pesquisa.

São Paulo, ....... de ................... de 2008 


\section{ANEXO II - $\underline{\text { Cronograma do experimento }}$}

\begin{tabular}{|c|c|c|c|c|c|c|c|}
\hline $\begin{array}{c}1 \text { semana } \\
\text { BA }\end{array}$ & $\begin{array}{c}2 \text { Feira 23/06 } \\
\text { Familiarização/AQ } \\
5 \text { tentativas }\end{array}$ & $\begin{array}{c}3 \text { Feira } 24 / 06 \\
\text { Familiarização/AQ } \\
5 \text { tentativas }\end{array}$ & $\begin{array}{c}4 \text { Feira } 25 / 06 \\
\text { AQ } \\
10 \text { tentativas }\end{array}$ & $\begin{array}{c}5 \text { Feira } 26 / 06 \\
\text { AQ } \\
10 \text { tentativas }\end{array}$ & $\begin{array}{c}6 \text { Feira } 27 / 06 \\
\text { AQ } \\
15 \text { tentativas }\end{array}$ & Sab 28/06 & Dom 29/06 \\
\hline $\begin{array}{c}2 \text { semana } \\
\text { BA }\end{array}$ & $\begin{array}{c}2 \text { Feira } 30 / 06 \\
\text { AQ } \\
15 \text { tentativas }\end{array}$ & $\begin{array}{c}3 \text { Feira } 01 / 07 \\
\text { AQ } \\
15 \text { tentativas }\end{array}$ & $\begin{array}{c}4 \text { Feira } 02 / 07 \\
\text { AQ } \\
15 \text { tentativas }\end{array}$ & $\begin{array}{c}5 \text { Feira } 03 / 07 \\
\text { AQ } \\
15 \text { tentativas }\end{array}$ & $\begin{array}{c}6 \text { Feira } 04 / 07 \\
\text { AQ } \\
15 \text { tentativas }\end{array}$ & Sab 05/07 & Dom $06 / 07$ \\
\hline $\begin{array}{c}3 \text { semana } \\
\text { BA }\end{array}$ & $\begin{array}{c}2 \text { Feira } 07 / 07 \\
\text { AQ } \\
15 \text { tentativas }\end{array}$ & $\begin{array}{c}3 \text { Feira } 08 / 07 \\
\mathrm{AQ} \\
15 \text { tentativas }\end{array}$ & $\begin{array}{c}4 \text { Feira } 09 / 07 \\
\text { AQ } \\
15 \text { tentativas }\end{array}$ & $\begin{array}{c}5 \text { Feira } 10 / 07 \\
\text { AQ } \\
15 \text { tentativas }\end{array}$ & $\begin{array}{c}6 \text { Feira } 11 / 07 \\
\text { AQ } \\
\text { Tentativas complementares } \\
\end{array}$ & Sab 12/07 & Dom $13 / 07$ \\
\hline $\begin{array}{c}4 \text { semana } \\
\text { BA }\end{array}$ & $\begin{array}{c}2 \text { Feira 14/07 } \\
\text { RT - TR }\end{array}$ & & & & & & \\
\hline $\begin{array}{c}1 \text { semana } \\
\mathrm{AL}\end{array}$ & $\begin{array}{c}2 \text { Feira } 04 / 08 \\
\text { Familiarizacão/AQ } \\
5 \text { tentativas } \\
\end{array}$ & $\begin{array}{c}\text { 3 Feira } 05 / 08 \\
\text { Familiarizacãa/AQ } \\
5 \text { tentativas }\end{array}$ & $\begin{array}{c}4 \text { Feira } 06 / 08 \\
\mathrm{AQ} \\
10 \text { tentativas }\end{array}$ & $\begin{array}{c}5 \text { Feira } 07 / 08 \\
\mathrm{AQ} \\
10 \text { tentativas } \\
\end{array}$ & $\begin{array}{c}6 \text { Feira } 08 / 08 \\
\text { AQ } \\
15 \text { tentativas } \\
\end{array}$ & Sab 09/08 & Dom $10 / 08$ \\
\hline $\begin{array}{c}2 \text { semana } \\
\mathrm{AL}\end{array}$ & $\begin{array}{c}2 \text { Feira } 11 / 08 \\
\text { AQ } \\
15 \text { tentativas }\end{array}$ & $\begin{array}{c}3 \text { Feira } 12 / 08 \\
\text { AQ } \\
15 \text { tentativas }\end{array}$ & $\begin{array}{c}4 \text { Feira } 13 / 08 \\
\text { AQ } \\
15 \text { tentativas }\end{array}$ & $\begin{array}{c}5 \text { Feira } 14 / 08 \\
\text { AQ } \\
15 \text { tentativas }\end{array}$ & $\begin{array}{c}6 \text { Feira } 15 / 08 \\
\text { AQ } \\
15 \text { tentativas }\end{array}$ & Sab 16/08 & Dom $17 / 08$ \\
\hline $\begin{array}{c}3 \text { semana } \\
\mathrm{AL}\end{array}$ & $\begin{array}{c}2 \text { Feira } 18 / 08 \\
\mathrm{AQ} \\
15 \text { tentativas } \\
\end{array}$ & $\begin{array}{c}3 \text { Feira } 19 / 08 \\
\text { AQ } \\
15 \text { tentativas }\end{array}$ & $\begin{array}{c}4 \text { Feira } 20 / 08 \\
\text { AQ } \\
15 \text { tentativas } \\
\end{array}$ & $\begin{array}{c}5 \text { Feira } 21 / 08 \\
\text { AQ } \\
15 \text { tentativas } \\
\end{array}$ & $\begin{array}{c}6 \text { Feira } 22 / 08 \\
\mathrm{AQ} \\
\text { Tentativas complementares } \\
\end{array}$ & Sab 23/08 & Dom $24 / 08$ \\
\hline $\begin{array}{c}4 \text { semana } \\
\mathrm{AL}\end{array}$ & $\begin{array}{c}2 \text { Feira } 25 / 08 \\
\text { RT - TR }\end{array}$ & & & & & & \\
\hline $\begin{array}{c}1 \text { semana } \\
\mathrm{CO} 1\end{array}$ & 2 Feira $01 / 09$ & 3 Feira $02 / 09$ & 4 Feira $03 / 09$ & 5 Feira 04/09 & 6 Feira $05 / 09$ & Sab 06/09 & Dom $07 / 09$ \\
\hline $\begin{array}{c}2 \text { semana } \\
\mathrm{CO} 1\end{array}$ & 2 Feira $08 / 09$ & 3 Feira $09 / 09$ & 4 Feira 10/09 & 5 Feira $11 / 09$ & 6 Feira $12 / 09$ & Sab 13/09 & Dom 14/09 \\
\hline $\begin{array}{c}3 \text { semana } \\
\mathrm{CO} 1\end{array}$ & 2 Feira $15 / 09$ & 3 Feira 16/09 & 4 Feira $17 / 09$ & 5 Feira $18 / 09$ & 6 Feira 19/09 & Sab 20/09 & Dom 21/09 \\
\hline $\begin{array}{c}4 \text { semana } \\
\mathrm{CO} 1\end{array}$ & $\begin{array}{c}2 \text { Feira } 22 / 09 \\
\text { Familiarização } \\
\text { RT - TR }\end{array}$ & $\begin{array}{c}\text { 3 Feira 23/09 } \\
\text { Familiarização } \\
\text { RT - TR }\end{array}$ & & & & & \\
\hline
\end{tabular}

\begin{tabular}{|c|c|c|}
\hline \multicolumn{3}{|l|}{ BA } \\
\hline Familiarização & 2 & dias \\
\hline $\mathrm{AQ}$ & 13 & dias \\
\hline $\mathrm{RT} / \mathrm{TR}$ & 1 & dias \\
\hline JANELAS & 6 & dias \\
\hline SEMANAS & 3 e 1 & dias \\
\hline TOTAL & 22 & dias \\
\hline \multicolumn{3}{|l|}{ BA } \\
\hline Familiarização & 2 & dias \\
\hline $\mathrm{AQ}$ & 13 & dias \\
\hline $\mathrm{RT} / \mathrm{TR}$ & 1 & dias \\
\hline JANELAS & 6 & dias \\
\hline SEMANAS & 3 e 1 & dias \\
\hline TOTAL & 22 & dias \\
\hline \multicolumn{3}{|l|}{$\mathrm{CO} 1$} \\
\hline AQ - (latência p/RT-TR) & 15 & dias \\
\hline Familiarização - RT/TR & 2 & dias \\
\hline JANELAS & 6 & dias \\
\hline SEMANAS & 3 e 2 & dias \\
\hline TOTAL & 23 & Dias \\
\hline
\end{tabular}


ANEXO III - Resultados da análise de variância de tempo de movimento

Resultados da ANOVA 2 (grupo) x 5 (bloco $[1,5,10,15,20]$ ) da fase de prática.

\begin{tabular}{|c|c|c|c|c|c|c|}
\hline \multicolumn{7}{|c|}{ FASE DE AQUISIÇÃO } \\
\hline \multicolumn{7}{|c|}{ 1-GRUPO, 2-BLOCO } \\
\hline & $\mathrm{gl}$ & MS & $\mathrm{gl}$ & MS & & \\
\hline & Efeito & Efeito & Erro & Erro & $\mathrm{F}$ & $\mathrm{p}$ \\
\hline 1 & 1 & 649,9440 & 18 & 235,0168 & 2,7655 & 0,1136 \\
\hline 2 & 4 & 4736,0884 & 72 & 120,2638 & 39,3808 & 0,0000 \\
\hline 12 & 4 & 1034,0605 & 72 & 120,2638 & 8,5983 & 0,0000 \\
\hline
\end{tabular}

Resultados das comparações post hoc.

\begin{tabular}{|c|c|c|c|c|c|c|c|c|c|}
\hline \multicolumn{10}{|c|}{ FASE DE AQUISIÇÃO } \\
\hline \multicolumn{10}{|c|}{ Probabilidade de teste post hoc } \\
\hline \multicolumn{10}{|c|}{ INTERAÇÃO: 1 x 2} \\
\hline $\mathrm{AL}\{1\}$ & $\operatorname{AL} 2\{2\}$ & $\operatorname{AL} 3\{3\}$ & $\operatorname{AL} 4\{4\}$ & $\mathrm{AL} 5\{5\}$ & BA $1\{6\}$ & BA $2\{7\}$ & BA $3\{8\}$ & BA $4\{9\}$ & BA $5\{10\}$ \\
\hline \multirow[t]{10}{*}{63.1070} & 16.9740 & 13.7320 & 11.6100 & 10.4370 & 32.3350 & 19.5600 & 13.7790 & 12.6740 & 12.0180 \\
\hline & 0,0001 & 0,0001 & 0,0001 & 0,0002 & 0,0001 & 0,0001 & 0,0001 & 0,0001 & 0,0001 \\
\hline & & 0,7868 & 0,8824 & 0,8342 & 0,0071 & 0,5997 & 0,5170 & 0,8169 & 0,8498 \\
\hline & & & 0,9727 & 0,9619 & 0,0029 & 0,6362 & 0,9925 & 0,8299 & 0,9350 \\
\hline & & & & 0,8118 & 0,0018 & 0,6694 & 0,9920 & 0,9745 & 0,9340 \\
\hline & & & & & 0,0010 & 0,5821 & 0,9835 & 0,9683 & 0,9445 \\
\hline & & & & & & 0,0113 & 0,0019 & 0,0021 & 0,0018 \\
\hline & & & & & & & 0,4697 & 0,6271 & 0,6414 \\
\hline & & & & & & & & 0,9725 & 0,9841 \\
\hline & & & & & & & & & 0,8941 \\
\hline
\end{tabular}

Resultados da ANOVA 3 (grupo) x 3 (teste).

\begin{tabular}{|l|r|c|r|c|r|c|}
\hline \multicolumn{7}{|c|}{ FASE DE RETENÇÃO E TRANSFERÊNCIA } \\
\hline 1-GRUPO, 2-TESTE \\
\hline & \multicolumn{1}{|c|}{$\mathrm{gl}$} & \multicolumn{1}{c|}{ MS } & \multicolumn{1}{c|}{$\mathrm{gl}$} & MS & & \\
\hline & Efeito & Efeito & Erro & Erro & F & p \\
\hline 1 & 2,00 & 20772,5898 & 27 & 820,6497 & 25,3124 & 0,0000 \\
\hline 2 & 2,00 & 5411,2295 & 54 & 367,6331 & 14,7191 & 0,0000 \\
\hline 12 & 4,00 & 924,5511 & 54 & 367,6331 & 2,5149 & 0,0520 \\
\hline
\end{tabular}


ANEXO IV - Resultados da análise de variância de variabilidade tempo de movimento

Resultados da ANOVA 2 (grupo) x 5 (bloco $[1,5,10,15,20]$ ) da fase de prática.

\begin{tabular}{|c|c|c|c|c|c|c|}
\hline \multicolumn{7}{|c|}{ FASE DE AQUISIÇÃO } \\
\hline \multicolumn{7}{|c|}{ 1-GRUPO, 2-BLOCO } \\
\hline & g1 & MS & $\mathrm{gl}$ & MS & & \\
\hline & Efeito & Efeito & Erro & Erro & $\mathrm{F}$ & $\mathrm{p}$ \\
\hline 1 & 1 & 763,9696 & 18 & 212,1518 & 3,601052 & 0,0739 \\
\hline 2 & 4 & 2616,3318 & 72 & 194,5304 & 13,44948 & 0,0000 \\
\hline 12 & 4 & 752,9678 & 72 & 194,5304 & 3,870695 & 0,0066 \\
\hline
\end{tabular}

Resultados das comparações post hoc.

\begin{tabular}{|c|c|c|c|c|c|c|c|c|c|}
\hline \multicolumn{10}{|c|}{ FASE DE AQUISIÇÃO } \\
\hline \multicolumn{10}{|c|}{ Probabilidade de teste post hoc } \\
\hline \multicolumn{10}{|c|}{ INTERAÇÃO: 1 x 2} \\
\hline $\mathrm{AL}\{1\}$ & $\operatorname{AL} 2\{2\}$ & $\operatorname{AL} 3\{3\}$ & $\operatorname{AL} 4\{4\}$ & $\mathrm{AL} 5\{5\}$ & BA $1\{6\}$ & BA $2\{7\}$ & BA $3\{8\}$ & BA $4\{9\}$ & BA $5\{10\}$ \\
\hline \multirow[t]{10}{*}{41.3930} & 2.75400 & 2.59600 & 1.72100 & 1.47900 & 13.9250 & 3.21700 & 1.91700 & 1.72300 & 1.52100 \\
\hline & 0,0002 & 0,0001 & 0,0001 & 0,0002 & 0,0001 & 0,0001 & 0,0001 & 0,0001 & 0,0001 \\
\hline & & 0,9800 & 0,9998 & 1,0000 & 0,1799 & 0,9411 & 0,9902 & 0,9984 & 1,0000 \\
\hline & & & 0,9991 & 1,0000 & 0,27427 & 0,9946 & 0,9137 & 0,9893 & 0,9998 \\
\hline & & & & 0,9993 & 0,45094 & 0,9999 & 0,9995 & 0,9998 & 0,9746 \\
\hline & & & & & 0,55166 & 1,0000 & 1,0000 & 1,0000 & 0,9947 \\
\hline & & & & & & 0,0905 & 0,3137 & 0,3775 & 0,4967 \\
\hline & & & & & & & 0,9968 & 0,9993 & 1,0000 \\
\hline & & & & & & & & 0,9754 & 0,9999 \\
\hline & & & & & & & & & 0,9995 \\
\hline
\end{tabular}

Resultados da ANOVA 3 (grupo) x 3 (teste).

\begin{tabular}{|l|r|c|r|r|r|r|}
\hline \multicolumn{7}{|c|}{ FASE DE RETENÇÃO E TRANSFERÊNCIA } \\
\hline 1-GRUPO, 2-TESTE \\
\hline & \multicolumn{1}{|c|}{$\mathrm{gl}$} & \multicolumn{1}{c|}{ MS } & \multicolumn{1}{c|}{$\mathrm{gl}$} & \multicolumn{1}{c|}{ MS } & & \\
\hline & Efeito & Efeito & \multicolumn{1}{c|}{ Erro } & Erro & F & $\mathrm{p}$ \\
\hline 1 & 2 & 3791,9561 & 27 & 107,9486 & 35,1274 & 0,0000 \\
\hline 2 & 2 & 868,2301 & 54 & 60,4312 & 14,3673 & 0,0000 \\
\hline 12 & 4 & 138,8416 & 54 & 60,4312 & 2,2975 & 0,0707 \\
\hline
\end{tabular}

